Bull. Korean Math. Soc. 50 (2013), No. 5, pp. 1523-1530

http://dx.doi.org/10.4134/BKMS.2013.50.5.1523

\title{
IDEALIZATIONS OF PSEUDO BUCHSBAUM MODULES OVER A PSEUDO BUCHSBAUM RING
}

\author{
Nguyen Thi Hong LoAn And Nong Quoc Chinh
}

\begin{abstract}
Let $(A, \mathfrak{m})$ be a Noetherian local ring and $M$ a finitely generated $A$-module. The notion of pseudo Buchsbaum module was introduced in [3] as an extension of that of Buchsbaum module. In this paper, we give a condition for the idealization $A \ltimes M$ of $M$ over $A$ to be pseudo Buchsbaum.
\end{abstract}

\section{Introduction}

Throughout this paper, let $(A, \mathfrak{m})$ be a Noetherian local ring and $M$ a finitely generated $A$-module with $\operatorname{dim} M=d$. The concept of principle of idealization was introduced by M. Nagata [10]. We make the Cartesian product $A \times M$ become a commutative ring under the componentwise addition and the multiplication defined by $(a, x)(b, y)=(a b, a y+b x)$. This ring is called the idealization of $M$ over $A$ and denoted by $A \ltimes M$. Note that the idealization $A \ltimes M$ is again a Noetherian local ring with the unique maximal ideal $\mathfrak{m} \times M$. The Krull dimension of $A \ltimes M$ equals $\operatorname{dim} A$.

The notion of principle of idealization plays an important role in the study of Noetherian rings and modules. For example, Reiten [11] used the principle of idealization to show that any Noetherian local ring possessing a Gorenstein module of rank 1 is a homomorphic image of a Gorenstein local ring. Then, Aoyama [1] studied the condition for the idealization to be quasi-Gorenstein and used this for the first step of the proof that any localization of the canonical module $K_{A}$ of $A$ at $\mathfrak{p} \in \operatorname{Supp}_{A} K_{A}$ is the canonical module of the local ring. In [13], K. Yamagishi clarified the condition for the idealization of Buchsbaum rings and modules to be Buchsbaum. Recently, Cuong-Morales-Nhan [6] used successively the notion of idealization in order to answer an open question by Sharp-Hamieh [12] on the polynomial property of the length of fractions.

The class of pseudo Buchsbaum modules was introduced by N. T. Cuong and the first author in [3], which contains strictly all Buchsbaum modules. The

Received May 14, 2012.

2010 Mathematics Subject Classification. 13E05, 13C14.

Key words and phrases. Buchsbaum modules, pseudo Buchsbaum modules, idealization. 
structure of pseudo Buchsbaum modules are known by the works of CuongLoan [3], [4], in which they presented many properties of these modules which are still good and closely related to that of Buchsbaum modules. Especially, if the ring $A$ admits a dualizing complex then $M$ is pseudo Buchsbaum if and only if the unmixed part $M / U_{M}(0)$ is Buchsbaum. Here $U_{M}(0)$ is the largest submodule of $M$ of dimension strictly less than $d$. The study of pseudo Buchsbaumness for Noetherian local rings is also very important because of the fact that the Monomial Conjecture raised by M. Hochster [9] is valid for all pseudo Buchsbaum rings.

In this paper, we study the pseudo Buchsbaumness for the idealization of finitely generated modules over a pseudo Buchsbaum ring. The following theorem is the main result of this paper.

Main Theorem. Let $A$ be a pseudo Buchsbaum ring and $M$ be an A-module with $\operatorname{dim} M=\operatorname{dim} A$. Then the following statements are true.

(i) If $A \ltimes M$ is pseudo Buchsbaum and $\operatorname{Ann}_{R} M=0$, then $M$ is pseudo Buchsbaum.

(ii) If $M$ is pseudo Buchsbaum with

$$
\sum_{i=1}^{d}\left(a_{1}^{t+1}, \ldots, a_{i-1}^{t+1}, a_{i+1}^{t+1}, \ldots, a_{d}^{t+1}\right) M:_{M} a_{i}=\left(a_{1}^{t+1}, \ldots, a_{d}^{t+1}\right) M
$$

for every system of parameters $\underline{a}=\left(a_{1}, \ldots, a_{d}\right)$ of $A$ and all large integers $t$, then $A \ltimes M$ is pseudo Buchsbaum.

In the next section, we recall some properties of pseudo Buchsbaum modules. The proof of Main Theorem will be shown in Section 3.

\section{Preliminaries}

In this section, we recall the notion of pseudo Buchsbaum modules and present some known properties of these modules that we need in the sequel.

Let $\underline{a}=\left(a_{1}, \ldots, a_{d}\right)$ be a system of parameters (s.o.p. for short) of $M$. Set

$$
Q_{M}(\underline{a})=\bigcup_{t>0}\left(a_{1}^{t+1}, \ldots, a_{d}^{t+1}\right) M:_{M} a_{1}^{t} \cdots a_{d}^{t} .
$$

For a $d$-tuple of positive integers $\underline{n}=\left(n_{1}, \ldots, n_{d}\right)$, put $\underline{a}(\underline{n})=\left(a_{1}^{n_{1}}, \ldots, a_{d}^{n_{d}}\right)$. Set

$$
J_{M}(\underline{a}(\underline{n}))=n_{1} \cdots n_{d} e(\underline{a} ; M)-\ell\left(M / Q_{M}(\underline{a}(\underline{n}))\right),
$$

where $e(\underline{a} ; M)$ is the multiplicity of $M$ with respect to $\underline{a}$. Then $J_{M}(\underline{a}(\underline{n}))$ can be considered as a function in $\underline{n}$. Note that this function is non-negative ([5, Lemma 3.1]) and ascending, i.e., $J_{M}(\underline{a}(\underline{n})) \geq J_{M}(\underline{a}(\underline{m}))$ whenever $n_{i} \geq$ $m_{i}$ for all $i=1, \ldots, d$, cf. [2, Corollary 4.3]. Sharp and Hamieh [12] asked that if the length of generalized fraction $1 /\left(a_{1}^{n_{1}}, \ldots, a_{d}^{n_{d}}, 1\right)$ is a polynomial for $\underline{n} \gg 0$, or equivalently, if the function $J_{M}(\underline{a}(\underline{n}))$ is a polynomial for $\underline{n} \gg 0$. Unfortunately, Cuong-Morales-Nhan [6] gave a counterexample to show that, 
in general $J_{M}(\underline{a}(\underline{n}))$ is not a polynomial for $\underline{n} \gg 0$. However, we have the following important result, cf. [5, Theorem 3.2].

Lemma 2.1. $J_{M}(\underline{a}(\underline{n}))$ is bounded from above by polynomials and the least degree of all polynomials bounding above this function is independent of the choice of the s.o.p. $\underline{a}$ of $M$.

Following Cuong-Minh [5], this least degree is denoted by $p f(M)$. The notions of pseudo Cohen-Macaulay module and pseudo Buchsbaum module were introduced by Cuong-Nhan [7] and Cuong-Loan [3] respectively. From now on, we stipulate that the degree of the zero-polynomial is $-\infty$.

Definition 2.2. $M$ is called pseudo Cohen-Macaulay if $p f(M)=-\infty$. We say that $M$ is pseudo Buchsbaum if there exists a constant $C$ such that $J_{M}(\underline{a})=C$ for every s.o.p $\underline{a}$ of $M$.

It should be mentioned that every Cohen-Macaulay (resp. Buchsbaum) module is pseudo Cohen-Macaulay (resp. pseudo Buchsbaum). The converse statement is not true. However, the following results, proved in [7] and [3], showed that the pseudo Cohen-Macaulayness (resp. pseudo Buchsbaumness) are closely related to the Cohen-Macaulayness (resp. Buchsbaumness).

From now on, denote by $U_{M}(0)$ the largest submodule of $M$ of dimension less than $d$. Set $\bar{M}=M / U_{M}(0)$ and $\widetilde{M}=\widehat{M} / U_{\widehat{M}}(0)$.

Lemma 2.3. Suppose that $A$ admits a dualizing complex. Then

(i) $M$ is pseudo Cohen-Macaulay if and only if $\bar{M}$ is Cohen-Macaulay.

(ii) $M$ is pseudo Buchsbaum if and only if $\bar{M}$ is Buchsbaum. In this case, for every s.o.p. $\underline{a}$ of $M$ we have

$$
J_{M}(\underline{a})=\sum_{i=1}^{d-1}\left(\begin{array}{l}
d-1 \\
i-1
\end{array}\right) \ell\left(H_{\mathfrak{m}}^{i}(\bar{M})\right) .
$$

Note that $p f(M)=p f(\widehat{M})$, cf. [5]. Hence $M$ is pseudo Cohen-Macaulay if and only if so is $\widehat{M}$. Therefore we get by Lemma 2.3 that $M$ is pseudo CohenMacaulay if and only if $\widetilde{M}$ is Cohen-Macaulay. The similar result for pseudo Buchsbaumness is also true, although its proof is much more complex (see [3]).

Lemma 2.4. $M$ is pseudo Buchsbaum if and only if $\widetilde{M}$ is Buchsbaum. In this case, for every s.o.p. $\underline{\text { a }}$ of $M$ we have

$$
J_{M}(\underline{a})=\sum_{i=1}^{d-1}\left(\begin{array}{l}
d-1 \\
i-1
\end{array}\right) \ell\left(H_{\widehat{\mathfrak{m}}}^{i}(\widetilde{M})\right) .
$$

The Monomial Conjecture raised by M. Hochster [9] says that for every s.o.p. $\underline{a}=\left(a_{1}, \ldots, a_{d}\right)$ of $A,\left(a_{1} \cdots a_{d}\right)^{t} \notin\left(a_{1}{ }^{t+1}, \ldots, a_{d}{ }^{t+1}\right) A$ for all positive integers $t$. It is well known that the Monomial Conjecture is always true for all Buchsbaum rings. The following result, which is proved in [3], claims that Monomial Conjecture is still valid for all pseudo Buchsbaum rings. 
Proposition 2.5. If $A$ is a pseudo Buchsbaum ring, then A satisfies the Monomial Conjecture.

\section{Proof of Main Theorem}

Firstly we recall the notion of principle of idealizations introduced by Nagata in [10]. We make the Cartesian product $A \times M$ into a commutative ring with respect to the componentwise addition and the multiplication defined by $(a, x)(b, y)=(a b, a y+b x)$. We call this ring to be the idealization of $M$ over $A$ and denote it by $A \ltimes M$. The idealization $A \ltimes M$ is a Noetherian local ring with the identity $(1,0)$, its unique maximal ideal is $\mathfrak{m} \times M$, and its Krull dimension is $\operatorname{dim} A$.

Remark 3.1. There is a canonical projection $\rho: A \ltimes M \rightarrow A$ defined by $\rho((a, x))$ $=a$ and a canonical inclusion $\sigma: A \rightarrow A \ltimes M$ defined by $\sigma(a)=(a, 0)$. These maps are local homomorphisms and we can regard any $A$-module (resp. $A \ltimes M$ module) as an $A \ltimes M$-module (resp. $A$-module) by $\rho$ (resp. $\sigma$ ). Moreover, the structure of $A$-modules induced by the composition $\rho \sigma$ coincides with the original one. Let $\epsilon: M \rightarrow A \ltimes M$ be the canonical inclusion defined by $\epsilon(x)=$ $(0, x)$. Then we have an exact sequence of $A \ltimes M$-modules

$$
0 \rightarrow M \rightarrow A \ltimes M \rightarrow A \rightarrow 0 .
$$

From now on, we assume that $\operatorname{dim} M=\operatorname{dim} A=d$.

Lemma 3.2. Let $\mathfrak{Q}$ be an ideal of $A \ltimes M$. Put $\mathfrak{q}:=\rho(\mathfrak{Q})$, where $\rho: A \ltimes M \rightarrow A$ is defined as in Remark 3.1. Then $\mathfrak{Q}$ is $\mathfrak{m} \times M$-primary if and only if $\mathfrak{q}$ is $\mathfrak{m}$-primary. In this case, we have

$$
e(\mathfrak{Q} ; A \ltimes M)=e(\mathfrak{q} ; A)+e(\mathfrak{q} ; M) .
$$

Proof. Since $\operatorname{dim} M=\operatorname{dim} A$, the result is clear by the above exact sequence.

Lemma 3.3. Let $\mathfrak{s}=\left(\left(a_{1}, x_{1}\right), \ldots,\left(a_{d}, x_{d}\right)\right)$ be a s.o.p. of the idealization ring $A \ltimes M$. Then $\underline{a}=\left(a_{1}, \ldots, a_{d}\right)$ is a s.o.p. of both $A$ and $M$. Let $\rho: A \ltimes M \rightarrow A$ and $\epsilon: M \rightarrow A \ltimes M$ be defined as in Remark 3.1. Then we have an exact sequence of $A \ltimes M$-modules

$$
M / Q_{M}(\underline{a}) \stackrel{\epsilon^{\prime}}{\rightarrow} A \ltimes M / Q_{A \ltimes M}(\mathfrak{s}) \stackrel{\rho^{\prime}}{\rightarrow} A / Q_{A}(\underline{a}) \rightarrow 0,
$$

where $\rho^{\prime}$ and $\epsilon^{\prime}$ are induced by $\rho$ and $\epsilon$, respectively.

Proof. We can check that $\rho\left(Q_{A \ltimes M}(\mathfrak{s})\right) \subseteq Q_{A}(\underline{a})$ and $\epsilon\left(Q_{A}(\underline{a})\right) \subseteq Q_{A \ltimes M}(\mathfrak{s})$. Now the result follows.

Lemma 3.4. Let $\mathfrak{s}=\left(\left(a_{1}, x_{1}\right), \ldots,\left(a_{d}, x_{d}\right)\right)$ be a s.o.p. of $A \ltimes M$. Suppose that

$$
\sum_{i=1}^{d}\left(a_{1}^{t+1}, \ldots, a_{i-1}^{t+1}, a_{i+1}^{t+1}, \ldots, a_{d}^{t+1}\right) M:_{M} a_{i}=\left(a_{1}^{t+1}, \ldots, a_{d}^{t+1}\right) M
$$


for all large integers $t(t \gg 0$ for short). Then

$$
(0 \times M) \cap Q_{A \ltimes M}(\mathfrak{s})=0 \times Q_{M}(\underline{a}) .
$$

Proof. Let $(0, m) \in 0 \times Q_{M}(\underline{a})$. Since $m \in Q_{M}(\underline{a})$, there exists some integer $t>0$ such that $m a_{1}^{t} \cdots a_{d}^{t} \in\left(a_{1}^{t+1}, \ldots, a_{d}^{t+1}\right) M$. So, we can write

$$
m a_{1}^{t} \cdots a_{d}^{t}=a_{1}^{t+1} y_{1}+\cdots+a_{d}^{t+1} y_{d}
$$

for some $y_{1}, \ldots, y_{d} \in M$. It is clear that $(a, x)^{t}=\left(a^{t}, t a^{t-1} x\right)$ for each element $(a, x) \in A \ltimes M$. Therefore we have

$$
(0, m)\left(a_{1}, x_{1}\right)^{t} \cdots\left(a_{d}, x_{d}\right)^{t}=\left(0, m a_{1}^{t} \cdots a_{d}^{t}\right)=\left(0, a_{1}^{t+1} y_{1}+\cdots+a_{d}^{t+1} y_{d}\right) .
$$

Hence $(0, m)\left(a_{1}, x_{1}\right)^{t} \cdots\left(a_{d}, x_{d}\right)^{t}=\left(a_{1}, x_{1}\right)^{t+1}\left(0, y_{1}\right)+\cdots+\left(a_{d}, x_{d}\right)^{t+1}\left(0, y_{d}\right)$ and hence

$$
(0, m)\left(a_{1}, x_{1}\right)^{t} \cdots\left(a_{d}, x_{d}\right)^{t} \in\left(\left(a_{1}, x_{1}\right)^{t+1}, \ldots,\left(a_{d}, x_{d}\right)^{t+1}\right)(A \ltimes M) .
$$

Therefore $(0, m) \in Q_{A \ltimes M}(\mathfrak{s})$. Thus, $0 \times Q_{M}(\underline{a}) \subseteq(0 \times M) \cap Q_{A \ltimes M}(\mathfrak{s})$.

Conversely, let $(0, m) \in(0 \times M) \cap Q_{A \ltimes M}(\mathfrak{s})$. Set $B=A \ltimes M$. Then we have $\left.\left(0, m a_{1}^{t} \cdots a_{d}^{t}\right)=(0, m)\left(a_{1}, x_{1}\right)^{t} \cdots\left(a_{d}, x_{d}\right)^{t}\right) \in\left(\left(a_{1}, x_{1}\right)^{t+1}, \ldots,\left(a_{d}, x_{d}\right)^{t+1}\right) B$ for all integers $t \gg 0$. Therefore there exist $\left(b_{1}, y_{1}\right), \ldots,\left(b_{d}, y_{d}\right) \in B$ such that

$$
\left(0, m a_{1}^{t} \cdots a_{d}^{t}\right)=\left(a_{1}, x_{1}\right)^{t+1}\left(b_{1}, y_{1}\right)+\cdots+\left(a_{d}, x_{d}\right)^{t+1}\left(b_{d}, y_{d}\right) .
$$

It follows that $a_{1}^{t+1} b_{1}+\cdots+a_{d}^{t+1} b_{d}=0$ and

$$
m a_{1}^{t} \cdots a_{d}^{t}=\left(a_{1}^{t+1} y_{1}+\cdots+a_{d}^{t+1} y_{d}\right)+t\left(a_{1}^{t} b_{1} x_{1}+\cdots+a_{d}^{t} b_{d} x_{d}\right) .
$$

For all $i=1, \ldots, d$, from the first equation we have

$$
a_{i}^{t+1} b_{i} \in\left(a_{1}^{t+1}, \ldots, a_{i-1}^{t+1}, a_{i+1}^{t+1}, \ldots, a_{d}^{t+1}\right) A .
$$

Hence $b_{i} \in\left(a_{1}^{t+1}, \ldots, a_{i-1}^{t+1}, a_{i+1}^{t+1}, \ldots, a_{d}^{t+1}\right) A: a_{i}^{t+1}$ and hence

$$
b_{i} x_{i} \in\left(a_{1}^{t+1}, \ldots, a_{i-1}^{t+1}, a_{i+1}^{t+1}, \ldots, a_{d}^{t+1}\right) M: a_{i}^{t+1}
$$

for all $i=1, \ldots, d$. Therefore $a_{i}^{t} b_{i} x_{i} \in\left(a_{1}^{t+1}, \ldots, a_{i-1}^{t+1}, a_{i+1}^{t+1}, \ldots, a_{d}^{t+1}\right) A: a_{i}$. It follows that

$$
\sum_{i=1}^{d} a_{i}^{t} b_{i} x_{i} \in \sum_{i=1}^{d}\left(a_{1}^{t+1}, \ldots, a_{i-1}^{t+1}, a_{i+1}^{t+1}, \ldots, a_{d}^{t+1}\right) A: a_{i}
$$

for all $i=1, \ldots, d$. By hypothesis we have $\sum_{i=1}^{d} a_{i}^{t} b_{i} x_{i} \in\left(a_{1}^{t+1}, \ldots, a_{d}^{t+1}\right) M$. Combining these facts, we get $m a_{1}^{t} \cdots a_{d}^{t} \in\left(a_{1}^{t+1}, \ldots, a_{d}^{t+1}\right) M$ for some integer $t \gg 0$. It means that $m \in Q_{M}(\underline{a})$. Hence $(0, m) \in 0 \times Q_{M}(\underline{a})$. Therefore

$$
(0 \times M) \cap Q_{A \ltimes M}(\mathfrak{s}) \subseteq 0 \times Q_{M}(\underline{a})
$$

and the result follows. 
Proof of Main Theorem. (i) Set $B=A \ltimes M$. Let $\underline{a}=\left(a_{1}, \ldots, a_{d}\right)$ be a s.o.p. of $M$. Since $\operatorname{Ann}_{R} M=0$ by hypothesis, it follows that $\left(a_{1}, \ldots, a_{d}\right) A$ is an $\mathfrak{m}$-primary ideal of $A$. Therefore $\underline{a}$ is a s.o.p. of $A$. By Lemma $3.2, \mathfrak{s}=$ $\left(\left(a_{1}, 0\right), \ldots,\left(a_{d}, 0\right)\right)$ is a s.o.p. of $B$. Therefore, we have by Lemma 3.3 an exact sequence of $B$-modules

$$
M / Q_{M}(\underline{a}) \stackrel{\epsilon^{\prime}}{\rightarrow} B / Q_{B}(\mathfrak{s}) \stackrel{\rho^{\prime}}{\rightarrow} A / Q_{A}(\underline{a}) \rightarrow 0 .
$$

For any element $(r, m) \in B$ and any integer $t>0$, we have

$$
\left(a_{1}, 0\right)^{t} \cdots\left(a_{d}, 0\right)^{t}(r, m)=\left(a_{1}^{t} \cdots a_{d}^{t} r, a_{1}^{t} \cdots a_{d}^{t} m\right) .
$$

Moreover,

$$
\left(\left(a_{1}, 0\right)^{t+1}, \ldots,\left(a_{d}, 0\right)^{t+1}\right) B=\left(a_{1}^{t+1}, \ldots, a_{d}^{t+1}\right) A \times\left(a_{1}^{t+1}, \ldots, a_{d}^{t+1}\right) M
$$

for any integer $t>0$. It follows that

$$
Q_{B}(\mathfrak{s})=Q_{A}(\underline{a}) \times Q_{M}(\underline{a}) .
$$

Then we get

$$
\text { Ker } \begin{aligned}
\epsilon^{\prime} & =\left\{m+Q_{M}(\underline{a}) \mid m \in M,(0, m) \in Q_{B}(\mathfrak{s})\right\} \\
& =\left\{m+Q_{M}(\underline{a}) \mid m \in M,(0, m) \in Q_{A}(\underline{a}) \times Q_{M}(\underline{a})\right\} \\
& =\left\{m+Q_{M}(\underline{a}) \mid m \in Q_{M}(\underline{a})\right\}=0 .
\end{aligned}
$$

Hence $\epsilon^{\prime}$ is injective and hence $\ell_{B}\left(B / Q_{B}(\mathfrak{s})\right)=\ell_{A}\left(M / Q_{M}(\underline{a})\right)+\ell_{A}\left(A / Q_{A}(\underline{a})\right)$. We have $e(\mathfrak{s} ; B)=e(\underline{a} ; M)+e(\underline{a} ; A)$ by Lemma 3.2. So, $J_{M}(\underline{a})=J_{B}(\mathfrak{s})-J_{A}(\underline{a})$. As $A$ and $B$ are pseudo Buchsbaum, so is $M$.

(ii) Let $\mathfrak{s}=\left(\left(a_{1}, x_{1}\right), \ldots,\left(a_{d}, x_{d}\right)\right)$ be a s.o.p. of $B$. Then $\underline{a}=\left(a_{1}, \ldots, a_{d}\right)$ is a s.o.p. of $A$ by Lemma 3.2. Therefore $\underline{a}$ is a s.o.p. of $M$. Then, we have by Lemma 3.3 an exact sequence of $B$-modules

$$
M / Q_{M}(\underline{a}) \stackrel{\epsilon^{\prime}}{\rightarrow} B / Q_{B}(\mathfrak{s}) \stackrel{\rho^{\prime}}{\rightarrow} A / Q_{A}(\underline{a}) \rightarrow 0 .
$$

By Lemma 3.4, we have $(0 \times M) \cap Q_{B}(\mathfrak{s})=0 \times Q_{M}(\underline{a})$. Therefore

$$
\begin{aligned}
\operatorname{Ker} \epsilon^{\prime} & =\left\{m+Q_{M}(\underline{a}) \mid m \in M,(0, m) \in Q_{B}(\mathfrak{s})\right\} \\
& =\left\{m+Q_{M}(\underline{a}) \mid m \in Q_{M}(\underline{a})\right\}=0 .
\end{aligned}
$$

So $\epsilon^{\prime}$ is injective. So, we get by the following exact sequence that

$$
\ell\left(B / Q_{B}(\mathfrak{s})\right)=\ell\left(M / Q_{M}(\underline{a})\right)+\ell\left(A / Q_{A}(\underline{a})\right) .
$$

Hence $J_{B}(\mathfrak{s})=J_{M}(\underline{a})+J_{A}(\underline{a})$ by Lemma 3.2. Because $A$ and $M$ are pseudo Buchsbaum, so is $B$.

Corollary 3.5. Suppose that $A$ admits a dualizing complex which is pseudo Buchsbaum. If $M$ is pseudo Cohen-Macaulay with $\operatorname{dim} A=\operatorname{dim} M=d$, then $A \ltimes M$ is pseudo Buchsbaum. 
Proof. Let $\underline{a}=\left(a_{1}, \ldots, a_{d}\right)$ be a s.o.p. of $A$. Then $\underline{a}$ is a s.o.p. of $M$. Let $U_{M}(0)$ be the largest submodule of $M$ of dimension less than $d$. Since $M$ is pseudo Cohen-Macaulay, we get by Lemma 2.3(i) that $\bar{M}=M / U_{M}(0)$ is Cohen-Macaulay. So, it follows by [3, Corollary 2.4] that

$$
\left[\left(a_{1}^{t+1}, \ldots, a_{d}^{t+1}\right) M+U_{M}(0)\right]: a_{1}^{t} \cdots a_{d}^{t}=\left(a_{1}, \ldots, a_{d}\right) M+U_{M}(0)
$$

for all integers $t \gg 0$. By [8, Theorem 2.3], the submodule

$$
\left[\left(a_{1}^{t+1}, \ldots, a_{d}^{t+1}\right) M+U_{M}(0)\right]: a_{1}^{t} \cdots a_{d}^{t}
$$

is equal to $\sum_{i=1}^{d}\left(\left[\left(a_{1}, \ldots, a_{i-1}, a_{i+1}, \ldots, a_{d}\right) M+U_{M}(0)\right]: a_{i}\right)$ for all integers $t \geq 1$. Therefore

$$
\sum_{i=1}^{d}\left[\left(a_{1}, \ldots, a_{i-1}, a_{i+1}, \ldots, a_{d}\right) M+U_{M}(0)\right]: a_{i}=\left(a_{1}, \ldots, a_{d}\right) M+U_{M}(0) .
$$

It follows that $\sum_{i=1}^{d}\left(a_{1}, \ldots, a_{i-1}, a_{i+1}, \ldots, a_{d}\right) M: a_{i}=\left(a_{1}, \ldots, a_{d}\right) M$ for any s.o.p. $\underline{a}$ of $M$. Now, the result follows by the Main Theorem.

Acknowledgment. The authors thank Professor Le Thanh Nhan for many useful suggestions.

\section{References}

[1] Y. Aoyama, Some basic results on canonical modules, J. Math. Kyoto Univ. 23 (1983), no. $1,85-94$.

[2] N. T. Cuong, N. T. Hoa, and N. T. H. Loan, On certain length functions associated to a system of parameters in local rings, Vietnam J. Math. 27 (1999), no. 3, 259-272.

[3] N. T. Cuong and N. T. H. Loan, A characterization for pseudo Buchsbaum module, Japan. J. Math. 30 (2004), no. 1, 165-181.

[4] - A blowing-up characterization of pseudo Buchsbaum modules, Vietnam J. Math. 34 (2006), no. 4, 449-458.

[5] N. T. Cuong and N. D. Minh, Lengths of generalized fractions of modules having small polynomial type, Math. Proc. Cambridge Philos. Soc. 128 (2000), no. 2, 269-282.

[6] N. T. Cuong, M. Morales, and L. T. Nhan, On the length of generalized fractions, J. Algebra 265 (2003), no. 1, 100-113.

[7] N. T. Cuong and L. T. Nhan, Pseudo Cohen Macaulay and pseudo generalized Cohen Macaulay modules, J. Algebra 267 (2003), no. 1, 156-177.

[8] S. Goto and K. Yamagshi, The theory of unconditioned strong d-sequences and modules of finite local cohomology, Preprint, 1986.

[9] M. Hochster, Contraced ideals from integral extensions of regular rings, Nagoya Math. J. 51 (1973), 25-43.

[10] M. Nagata, Local Rings, Interscience Tracts in Pure and Applied Mathematics, No. 13 Interscience Publishers a division of John Wiley \& Sons New York-London 1962.

[11] I. Reiten, The converse to a theorem of Sharp on Gorenstein modules, Proc. Amer. Math. Soc. 32 (1972), 417-420.

[12] R. Y. Sharp and M. A. Hamieh, Lengths of certain generalized fractions, J. Pure Appl. Algebra 38 (1985), no. 2-3, 323-336.

[13] K. Yamagishi, Idealizations of maximal Buchsbaum modules over a Buchsbaum ring, Math. Proc. Cambridge Philos. Soc. 104 (1988), no. 3, 451-478. 
NGUyen Thi Hong LOAN

VINH UNIVERSITY

NGHE An, Vietnam

E-mail address: hongloanncs@yahoo.com

Nong Quoc Chinh

Thai Nguyen College of Sciences

Thai Nguyen, Vietnam

E-mail address: chinhnq2010@gmail.com 